\title{
Cospectral graphs on 12 vertices
}

\author{
A. E. Brouwer \\ Dept. of Mathematics \\ Techn. Univ. Eindhoven \\ P.O. Box 513, 5600MB Eindhoven \\ Netherlands \\ aeb@cwi.nl
}

\author{
E. Spence \\ Dept. of Mathematics \\ University of Glasgow \\ Glasgow G12 8QQ \\ Scotland \\ ted@maths.gla.ac.uk
}

Submitted: Jun 1, 2009; Accepted: Jun 2, 2009; Published: Jun 12, 2009

Mathematics Subject Classification: 05C50, 05E99

\begin{abstract}
We found the characteristic polynomials for all graphs on 12 vertices, and report statistics related to the number of cospectral graphs.

\section{Introduction}

Let the spectrum of a graph be the spectrum of its 0-1 adjacency matrix. In connection with the graph isomorphism problem, it is of interest what fraction of all graphs is uniquely determined by its spectrum. Haemers conjectures that the fraction of graphs on $n$ vertices with a cospectral mate tends to zero as $n$ tends to infinity. Numerical data for $n \leq 9$ was given in [2], and for $n=10,11$ in [3]. Here we do $n=12$, and also take the opportunity to correct a few earlier values.

Both authors did the computations independently and found the same results.
\end{abstract}

\section{Totals}

The table below lists for $n \leq 12$ the total number of graphs on $n$ vertices, the total number of distinct characteristic polynomials of such graphs, the number of such graphs with a cospectral mate, and the size of the largest family of cospectral graphs. 


\begin{tabular}{rrrrr} 
n & \#graphs & \#char. pols & \#with mate & max. family \\
\hline 0 & 1 & 1 & 0 & 1 \\
1 & 1 & 1 & 0 & 1 \\
2 & 2 & 2 & 0 & 1 \\
3 & 4 & 4 & 0 & 1 \\
4 & 11 & 11 & 0 & 1 \\
5 & 34 & 33 & 2 & 2 \\
6 & 156 & 151 & 10 & 2 \\
7 & 1044 & 988 & 110 & 3 \\
8 & 12346 & 11453 & 1722 & 4 \\
9 & 274668 & 247357 & $51039^{*}$ & 10 \\
10 & 12005168 & 10608128 & $2560606^{*}$ & 21 \\
11 & 1018997864 & 901029366 & $215331676^{*}$ & 46 \\
12 & 165091172592 & 148187993520 & 31067572481 & 128
\end{tabular}

The three starred entries are 1 more, 90 more, and 1 less than the corresponding values in [3]. (The first of these was given correctly in [2].)

\section{Trends}

In the table above we see that the fraction of graphs with a cospectral mate increases at first and starts decreasing at $n=11$. Graphically:

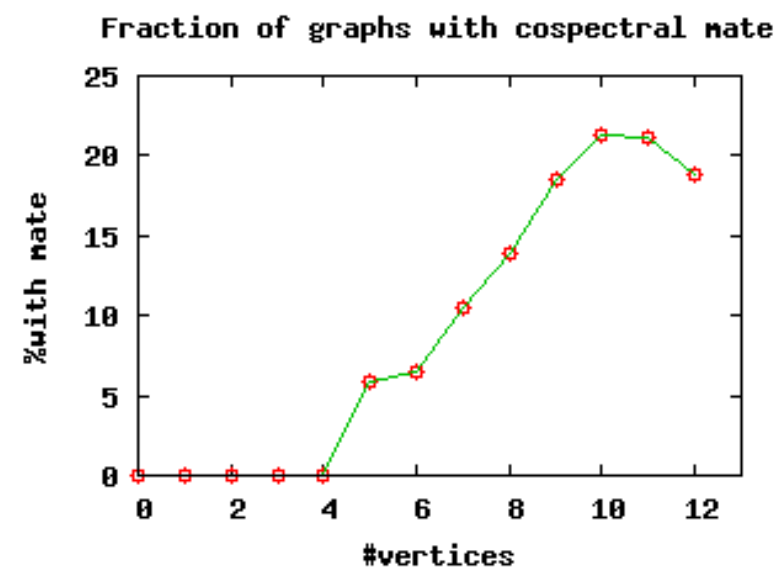

Somewhat more illuminating are the below plots for $n=9,10,11,12$ where the percentage of graphs with cospectral mate is given as function of the number of edges. One sees that the central part of the graph is pressed down as we go from $n=9$ to $n=12$, but the parts for low or high edge density might show some increase. For some more details, see [1]. 

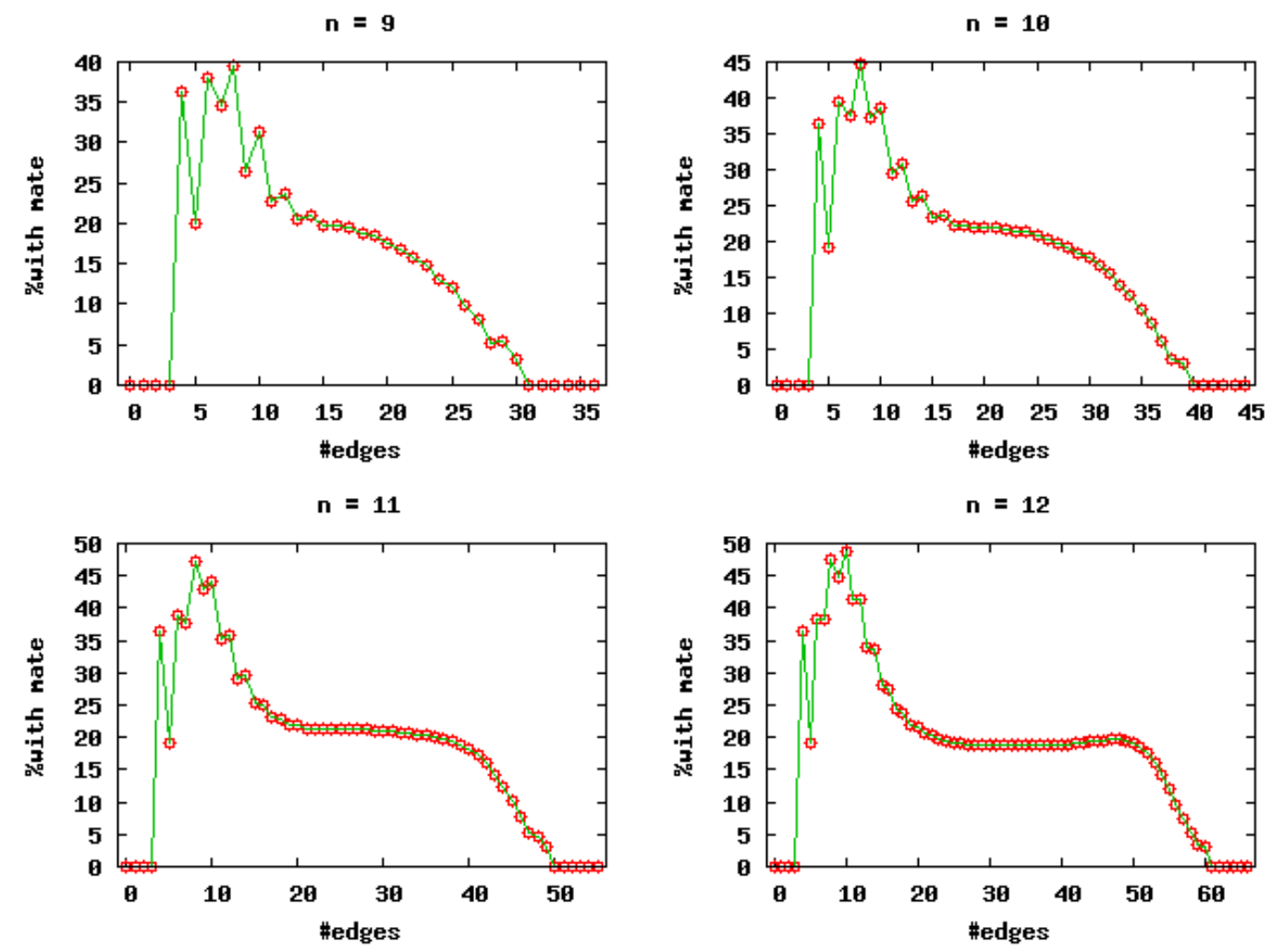

There is a clear odd-even effect.

\section{References}

[1] http://www.win.tue.nl/〜aeb/graphs/cospectral/cospectralA.html

[2] C. Godsil \& B. McKay, Some computational results on the spectra of graphs, in: Combinatorial Mathematics IV, Springer LNM 560 (1976) 73-92.

[3] W. H. Haemers \& E. Spence, Enumeration of cospectral graphs, Europ. J. Combin. 25 (2004) 199-211. 